\title{
Sangrado gastrointestinal secundario a síndrome de Klippel-Trenaunay
}

\author{
Gastrointestinal bleeding secondary to Klippel - Trenaunay syndrome
}

\author{
Manuel Alejandro Giraldo-Pinto ${ }^{1} \mathbb{D}$, Jessica Capre-Pereira ${ }^{2} \mathbb{D}$, Jairo González-Quitian ${ }^{1} \mathbb{D}$, \\ Abraham Kestenberg $^{3} \mathbb{D}$, Mónica Bejarano ${ }^{4}$ D
}

1 Médico, residente de Cirugía general, Universidad del Valle, Cali, Colombia.

2 Médica, especialista en Cirugía general, Departamento de Cirugía general, Fundación Valle del Lili; docente, Cirugia general, Universidad ICESI, Cali, Colombia.

3 Médica, especialista en Cirugía general, subespecialista en Cirugía de Colon y Recto, Fundación Valle del Lili, Cali, Colombia.

4 Médica, especialista en Cirugía general, magister en Epidemiología, Departamento de Cirugía general, Fundación Valle del Lili; docente, Cirugía general, Universidad ICESI, Cali, Colombia.

\section{Introducción}

El síndrome de Klippel-Trenaunay es una entidad poco frecuente, descrita en 1900 por dos médicos franceses ${ }^{1}$, con una incidencia estimada en 1 de cada 100.000 nacidos vivos ${ }^{2}$. Se caracteriza por la presencia de malformaciones arteriovenosas, de predominio en miembros inferiores, que generan hipertrofia en huesos y tejidos blandos ${ }^{2,3}$. Rara vez afecta el tracto gastrointestinal, pero, si lo hace, puede causar importante sangrado digestivo, llegando a comprometer la vida del paciente ${ }^{4}$. Presentamos el caso de un paciente con sangrado gastrointestinal secundario a síndrome de KlippelTrenaunay.

\section{Caso Clínico}

Se trata de un paciente masculino de 26 años, con antecedentes de hemangiomas y deformidad de dedos en miembro inferior derecho desde el nacimiento (Figura 1) y rectorragia tres años antes. Se realizaron estudios con los que identificaron múltiples varices en recto, con extensión a colon sigmoides, y trombosis de la vena mesentérica inferior, que generaba hipertensión portal. Se llevó a cirugía de derivación porto-cava, con mejoría completa del cuadro clínico en ese momento.

Consulta ahora por nuevos episodios de rectorragia, con descenso de hemoglobina hasta

Palabras Clave: Síndrome de Klippel-Trenaunay-Weber; hemorragia gastrointestinal; enfermedades raras; enfermedades del colon; malformaciones arteriovenosas.

Keywords: Klippel-Trenaunay-Weber Syndrome; gastrointestinal hemorrhage; rare diseases; colonic diseases; arteriovenous malformations.

Fecha de recibido: 29/06/2021 - Fecha de aceptación: 08/07/2021 - Fecha de publicación en línea: 06/08/2021

Autor de Correspondencia: Manuel Alejandro Giraldo-Pinto, Carrera 46 \# 8B - 95 Apto 1102, Cali, Colombia. Tel: 3146174945. Dirección electrónica: man_giraldo@hotmail.com

Citar como: Giraldo-Pinto MA, Capre-Pereira J, González-Quitian J, Kestenberg A, Bejarano M. Sangrado gastrointestinal secundario a síndrome de Klippel- Trenaunay. Rev Colomb Cir. 2021;36:709-11. https://doi.org/10.30944/20117582.981

Este es un artículo de acceso abierto bajo una Licencia Creative Commons - BY-NC-ND https://creativecommons.org/licenses/by-nc$\mathrm{nd} / 4.0 /$ deed.es 
2,2 g/dL. Se realizó angiotomografía abdominal que evidenció oclusión de derivación porto-cava y sangrado proveniente de arteria hemorroidal superior. Se practicó derivación porto-sistémica transyugular intrahepática y arteriografía donde se confirmó sangrado proveniente de la arteria hemorroidal superior, y fue tratado satisfactoriamente con técnica endovascular.

Dos días después presentó nuevo episodio de abundante sangrado rectal, por lo que se decidió llevar a cirugía, donde se encontró gran vasodilatación del colon sigmoides y recto, generando distensión e hiperemia (Figura 2). Se practicó resección del colon sigmoides y recto superior, con colostomía tipo Hartman.

Los hallazgos macroscópicos de la pieza quirúrgica fueron serosa violácea, altamente vascularizada, con vasos tortuosos y dilatados, y mucosa con similares características (Figura 3). En la histopatología de la pieza quirúrgica se identificaron múltiples espacios vasculares dilatados, con compromiso de todas las capas del colon, hallazgos compatibles con malformación arteriovenosa.

El paciente evoluciono satisfactoriamente logrando control completo del sangrado gastrointestinal.

\section{Discusión}

El síndrome de Klippel-Trenaunay no tiene criterios diagnósticos definidos. Se ha propuesto la

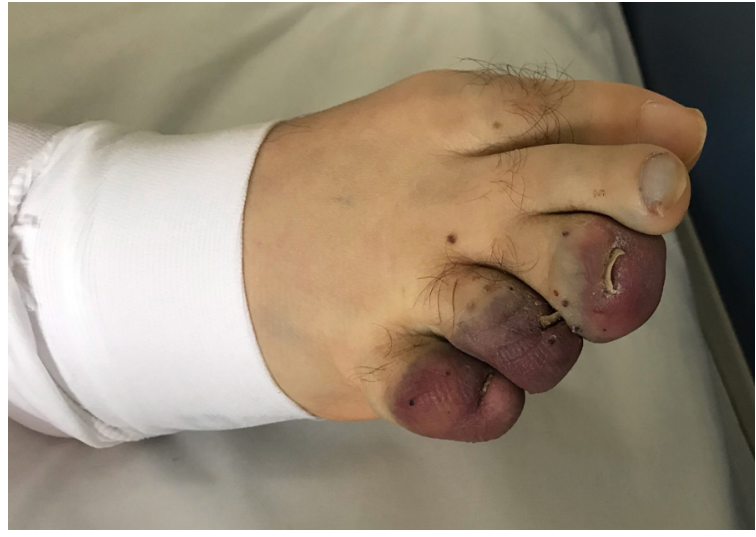

Figura 1. Hemangiomas y deformidad ósea de miembro inferior derecho. Fuente: Autores triada compuesta por hemangiomas cutáneos, varices e hipertrofia de hueso y tejidos blandos ${ }^{3}$. El compromiso gastrointestinal es raro (1-12,5 \% de los casos) ${ }^{5}$ y se manifiesta con sangrado digestivo, originado principalmente en el colon distal y el recto, que puede comprometer la vida ${ }^{6}$.

Por tratarse de una patología de muy baja incidencia, no existen consensos claramente establecidos, y el tratamiento consiste en el manejo de los síntomas, en la medida que se presentan ${ }^{2,3}$. Algunos casos pueden ser enfocados de manera conservadora; la terapia endovascular y la cirugía se reservan para aquellos pacientes que son dependientes de transfusiones sanguíneas ${ }^{4,5,6}$.

\section{Consideraciones éticas}

Consentimiento informado: Se obtuvo consentimiento informado del paciente para la publicación del caso y las fotografías, aunque las imágenes no permiten la identificación del paciente.

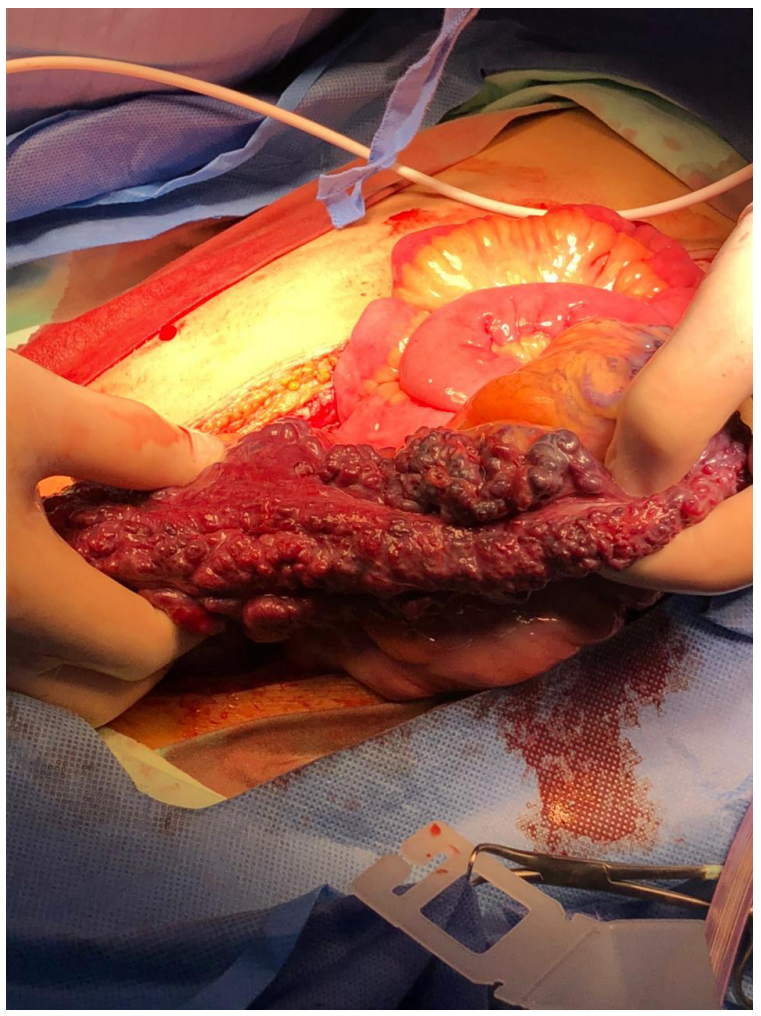

Figura 2. Dilataciones vasculares en colon sigmoides. Fuente: Autores 

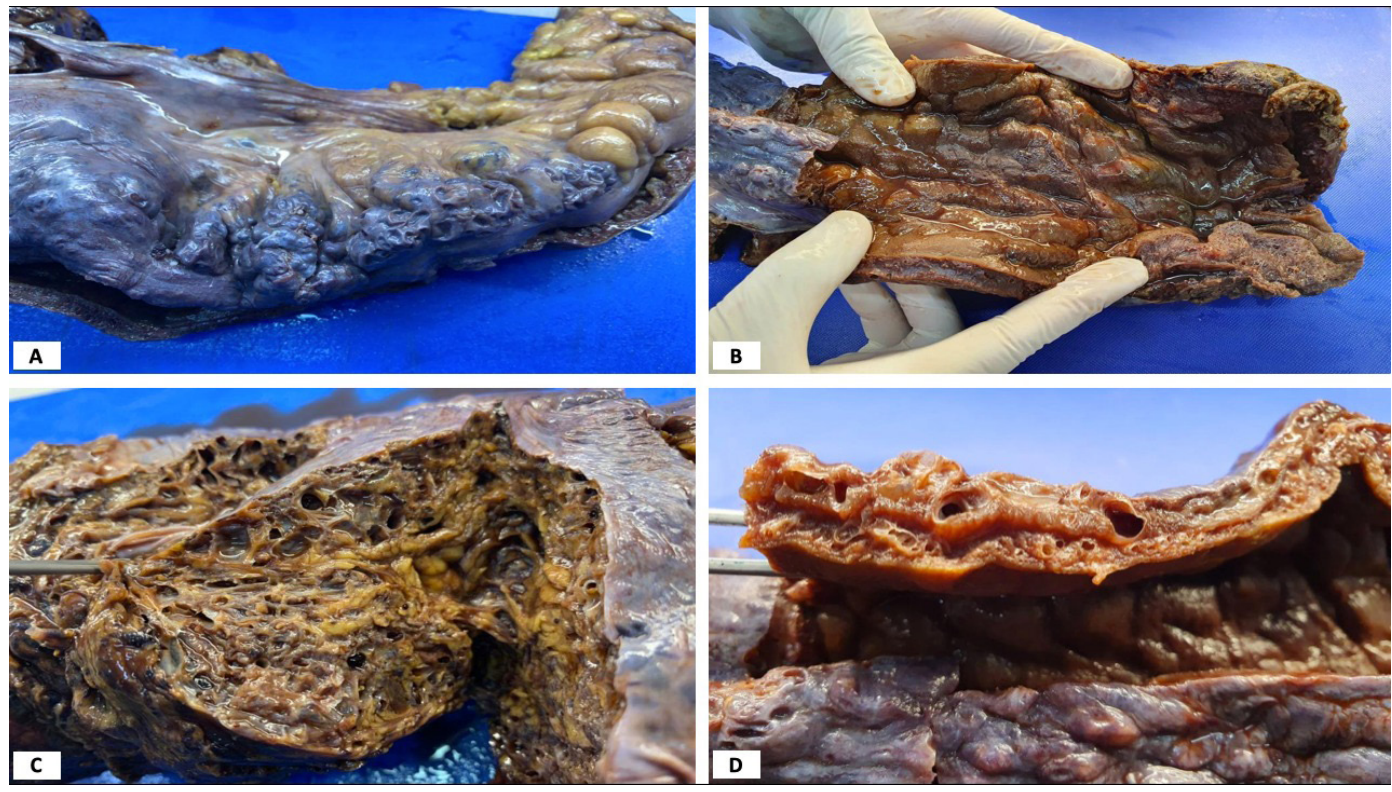

Figura 3. Pieza quirúrgica macroscópica de colon sigmoides y recto superior A. Serosa congestiva con gran dilatación de vasos sanguíneos $\mathrm{B}$. Mucosa con vasos sanguíneos dilatados y tortuosos $\mathrm{C}$ y D. Evidencia de compromiso descrito en todas las capas del colon. Fuente: Autores

Conflicto de intereses: los autores declararon no tener ningún conflicto de interés.

Fuente de financiación: recursos propios de los autores.

Contribución de los autores:

Concepción y diseño del estudio: Manuel Alejandro Giraldo-Pinto, Jessica Capre-Pereira, Jairo GonzálezQuitian, Abraham Kestenberg, Mónica Bejarano.

Adquisición de datos: Manuel Alejandro Giraldo-Pinto, Jessica Capre-Pereira, Jairo González-Quitian.

Análisis e interpretación de datos: Manuel Alejandro Giraldo-Pinto, Jessica Capre-Pereira, Abraham Kestenberg. Redacción del manuscrito: Manuel Alejandro GiraldoPinto, Mónica Bejarano.

Revisión crítica: Jessica Capre-Pereira, Abraham Kestenberg, Mónica Bejarano.

\section{Referencias}

1. Sharma D, Lamba S, Pandita A, Shastri S. Klippel-Trénaunay syndrome - A very rare and interesting syndrome. Clin Med Insights Circ Respir Pulm Med. 2015 Jan;9:CCRPM.S21645. http://dx.doi.org/10.4137/CCrpM.s21645
2. Espín G, Suntaxi L, Yambay C, Silva R, Espín L, Vásquez B. Síndrome congénito de Klippel-Trenaunay-Weber. Caso Clínico. Int J Morphol. 2020;38:1842-8. http://dx.doi.org/10.4067/S0717-9502202000 0601842

3. Asghar F, Aqeel R, Farooque U, Haq A, Taimur M. Presentation and management of Klippel-Trenaunay syndrome: A review of available data. Cureus. 2020;12:e8023. http://dx.doi.org/10.7759/cureus.8023

4. Then EO, Ofosu A, Rawla P, Sunkara T, Gaduputi V. Klippel-Trenaunay syndrome, a rare cause of hematochezia. Gastroenterol Res. 2018;11:426-9. http://dx.doi.org/10.14740/gr1092

5. Thosani N, Ghouri Y, Shah S, Reddy S, Arora G, Scott L. Life-threatening gastrointestinal bleeding in Klippel-Trenaunay syndrome. Endoscopy. 2013;45(S 02):E206-E206.

http://dx.doi.org/10.1055/s-0032-1326645

6. Wilson CL, Wong Kee Song L-M, Chua H, Ferrara M, Devine RM, Dozois RR, et al. Bleeding from cavernous angiomatosis of the rectum in Klippel-Trenaunay syndrome: report of three cases and literature review. Am J Gastroenterology. 2001;96:2783-8. http://dx.doi.org/10.1111/j.1572-0241.2001.04110.x 